\title{
The regulation of cell wall lignification and lignin biosynthesis during pigmentation of winter jujube
}

\author{
Qiong Zhang ${ }^{1}$, Lihu Wang $\mathbb{1}^{2}$, Zhongtang Wang ${ }^{1}$, Rentang Zhang ${ }^{3}$, Ping Liư ${ }^{4}$ Mengjun Liư ${ }^{4}$ Zhiguo Liư \\ Zhihui Zhao ${ }^{4}$, Lili Wang ${ }^{4}$, Xin Chen ${ }^{1 凶}$ and Haifeng $\mathrm{Xu}^{1 凶}$
}

\begin{abstract}
Fruit lignification is due to lignin deposition in the cell wall during cell development. However, there are few studies on the regulation of cell wall lignification and lignin biosynthesis during fruit pigmentation. In this study, we investigated the regulation of cell wall lignification and lignin biosynthesis during pigmentation of winter jujube. The cellulose content decreased, while the lignin content increased in the winter jujube pericarp during pigmentation. Safranin O-fast green staining showed that the cellulose content was higher in the cell wall of winter jujube prior to pigmentation, whereas the lignin in the cell wall increased after pigmentation. The thickness of the epidermal cells decreased with pericarp pigmentation. A combined metabolomics and transcriptomics analysis showed that guaiacyl-syringyl (G-S) lignin was the main lignin type in the pericarp of winter jujube, and F5H (LOC107424406) and CCR (LOC107420974) were preliminarily identified as the key genes modulating lignin biosynthesis in winter jujube. Seventeen MYB and six NAC transcription factors (TFs) with potential regulation of lignin biosynthesis were screened out based on phylogenetic analysis. Three MYB and two NAC TFs were selected as candidate genes and further studied in detail. Arabidopsis ectopic expression and winter jujube pericarp injection of the candidate genes indicated that the MYB activator (LOC107425254) and the MYB repressor (LOC107415078) control lignin biosynthesis by regulating CCR and F5H, while the NAC (LOC107435239) TF promotes $\mathrm{F} 5 \mathrm{H}$ expression and positively regulates lignin biosynthesis. These findings revealed the lignin biosynthetic pathway and associated genes during pigmentation of winter jujube pericarp and provide a basis for further research on lignin regulation.
\end{abstract}

\section{Introduction}

Jujube (Ziziphus jujuba Mill.) is a medicinal and edible fruit that originates from China. Owing to various characteristics, such as tolerance of barren environments, saltalkali conditions, drought, wind and sand, and early and quick harvesting, jujube trees are pioneers in promoting economic development and ecological construction in sand-alkali-arid regions ${ }^{1}$. Jujube fruit is nutritious and rich in a variety of functionally active components. Recent studies have found that ursolic acid and oleanolic acid in

\footnotetext{
Correspondence: Xin Chen (sdaucx@163.com) or

Haifeng Xu (xhfdl1991@163.com)

'Shandong Institute of Pomology, Tai'an, Shandong 271000, China

${ }^{2}$ School of Landscape and Ecological Engineering, Hebei University of Engineering, Handan, Hebei 056038, China

Full list of author information is available at the end of the article
}

jujube fruit extracts inhibit breast cancer metastasis and cell apoptosis ${ }^{2}$. Moreover, polyphenols in the jujube pericarp were reported to effectively protect against myocardial ischemia caused by isoproterenol ${ }^{3}$.

Lignin is an important component of cell walls that maintains the rigidity of cell walls and provides mechanical support for the cells. Its hydrophobicity can prevent water loss. The secondary lignification of cell walls plays a key role in the growth and development of plants, as well as in the resistance to various biotic and abiotic stresses ${ }^{4}$. Lignin contains three monomers, namely, $p$-hydroxyphenyl lignin ( $\mathrm{H}$ lignin), syringyl lignin (S lignin), and guaiacyl lignin ( $\mathrm{G}$ lignin). Lignin biosynthesis is one of the most important flavonoid biosynthesis pathways, while the $P A L, C 4 H$, and $4 C L$ genes serve as the common structural genes of the flavonoid

\section{(c) The Author(s) 2021}

\footnotetext{
(c) Open Access This article is licensed under a Creative Commons Attribution 4.0 International License, which permits use, sharing, adaptation, distribution and reproduction cc) in any medium or format, as long as you give appropriate credit to the original author(s) and the source, provide a link to the Creative Commons license, and indicate if changes were made. The images or other third party material in this article are included in the article's Creative Commons license, unless indicated otherwise in a credit line to the material. If material is not included in the article's Creative Commons license and your intended use is not permitted by statutory regulation or exceeds the permitted use, you will need to obtain permission directly from the copyright holder. To view a copy of this license, visit http://creativecommons.org/licenses/by/4.0/.
} 
and lignin biosynthesis pathways. Silencing of the $P A L$ gene in tobacco resulted in decreased flavonoid and lignin contents and impaired plant growth and development ${ }^{5}$. Knockout of the $\mathrm{C} 4 \mathrm{H}$ gene in Arabidopsis (Arabidopsis thaliana Heynh.) blocked the biosynthesis of flavonoid and lignin ${ }^{6}$. In addition, downregulating the expression of various genes, including cinnamoyl $\mathrm{CoA}$ reductase $(C C R)$, cinnamyl alcohol dehydrogenase $(C A D)$, and hydroxycinnamoyl-CoA $(H C T)$, in Arabidopsis reduced the lignin content but increased the flavonoid content, and the plants were smaller with stunted development ${ }^{7,8}$.

Lignin biosynthesis is regulated by MYB TFs. Some MYB TFs can simultaneously regulate flavonoid and lignin synthesis in positive or negative directions. For instance, CmMYB8 in chrysanthemum (Dendranthema morifolium Tzvel.) simultaneously represses the accumulation of lignin and flavonoids ${ }^{9}$. However, most MYB TFs can only positively regulate flavonoid or lignin synthesis. For example, the overexpression of tomato SIMYB4 significantly reduced the expression levels of structural genes such as $P A L, 4 C L, C 4 H$, and $C C R$ and decreased lignin accumulation. In contrast, there was no significant change in the flavonoid content since $S l M Y B 4$ could not bind to the SlCHS1 promoter ${ }^{10}$. The overexpression of grape $V v M Y B 5 a$ and $V v M Y B 5 b$ in petunia upregulated the expression of the $P A L, C 4 H$, and $4 C L$ genes, increased the content of anthocyanin, downregulated CCOAOMT1 expression, and reduced lignin content ${ }^{11}$. Furthermore, NAC is another specific TF in lignin biosynthesis. It has been reported that the fruit lignification of pear (Pyrus pyrifolia) is regulated by $P p N A C 187$. Specifically, the overexpression of $P p N A C 187$ upregulated the expression of the CCR and COMT genes and increased the content of lignin ${ }^{12}$. Another study showed that the overexpression of PdWND3A in poplar (Populus euphratica Oliv.) upregulated the expression of the $F 5 H 1$ gene and promoted the biosynthesis of lignin ${ }^{13}$.

The pigmentation process of the jujube pericarp occurs with the ripening of the fruit and is accompanied by the deposition of insoluble fiber in the cell walls. However, studies on the regulation of cell wall lignification and lignin biosynthesis in jujube are lacking. Herein, we analyzed the changes in insoluble fiber and pericarp cells and the regulation of lignin biosynthesis during pigmentation of winter jujube ( $Z$. jujuba Mill. cv. Dongzao) using biochemical, metabolomic, transcriptomic, and transgenic approaches.

\section{Results}

Changes in insoluble fiber contents and cell structures during the pigmentation process of winter jujube pericarp

The pericarp color of winter jujube fruit at different periods of maturation varies from white $(\mathrm{W})$ to semi-red
(SR) to full-red (R) (Fig. 1A). As shown in Fig. 1B, cellulose was the main component in the pericarp of winter jujube, followed by hemicellulose and lignin. The cellulose content gradually decreased from the $\mathrm{W}$ stage to the $\mathrm{R}$ stage. In contrast, the hemicellulose and lignin contents of the winter jujube pericarp were the lowest in the $\mathrm{W}$ stage and increased by 52 and $91 \%$ in the $R$ stage, respectively.

The epidermal cells of jujube were arranged closely and neatly at the W stage and SR-W stage, with oblate or oblong shapes (Fig. 1C). At the SR-R and $R$ stages, the epidermal cells of winter jujube were significantly shriveled with increased transverse diameters and decreased longitudinal diameters with oblong shapes. Moreover, the intercellular space was increased, and the arrangement was looser than that in the prepigmentation stage (Fig. 1C). As shown in Fig. 1D, there was no color in the unstained jujube pericarp cells, while overall pericarp cells and cell walls displayed orange-yellow and orangered colors, respectively, after pigmentation. Safranin O-fast green staining results (Fig. 1C) showed that the cell wall prior to pigmentation was blue-green in color with almost no color inside the cell. In contrast, the cells after pigmentation presented a red color, with an increased cell wall thickness. According to the principle of safranin O-fast green staining, safranin displayed the red color of the lignified cell wall, and fast green displayed the blue-green color of cellulose in the cell wall. Our results indicate that the cellulose content in the cell wall of winter jujube was high before pigmentation, while the lignin content was high when the cells became lignified after pigmentation.

As shown in Fig. 1E, the thickness of cuticle cells in the pericarp of winter jujube showed no significant change from the $\mathrm{W}$ to $\mathrm{R}$ stage, ranging from 9.3 to $10.2 \mu \mathrm{m}$. The thickness of the 5-6 layers of epidermal cells decreased with the pigmentation of the pericarp. Specifically, there was no significant change from the $\mathrm{W}$ stage $(83.6 \mu \mathrm{m})$ to the SR-W stage $(83.5 \mu \mathrm{m})$. However, there was a significant decrease to $59.7 \mu \mathrm{m}$ at the SR-R stage and $61.2 \mu \mathrm{m}$ at the $\mathrm{R}$ stage, demonstrating a decrease of $20 \mu \mathrm{m}$ in thickness after pigmentation.

\section{Observation of pigmentation on the pericarp of winter jujube}

Figure 2 shows the anatomical structure of frozen sections of winter jujube pericarp at the $\mathrm{R}$ stage with six different thicknesses $(5,10,15,20,25$, and $30 \mu \mathrm{m})$. Consistent with the results from the paraffin sections, the color of the jujube pericarp adhered to the cuticle cells and 5-6 layers of epidermal cells. The epidermal cells of the jujube pericarp were oblong, with a large intercellular space and cellular pigmentation. As the section thickness increased, the color gradually darkened from yellow to 


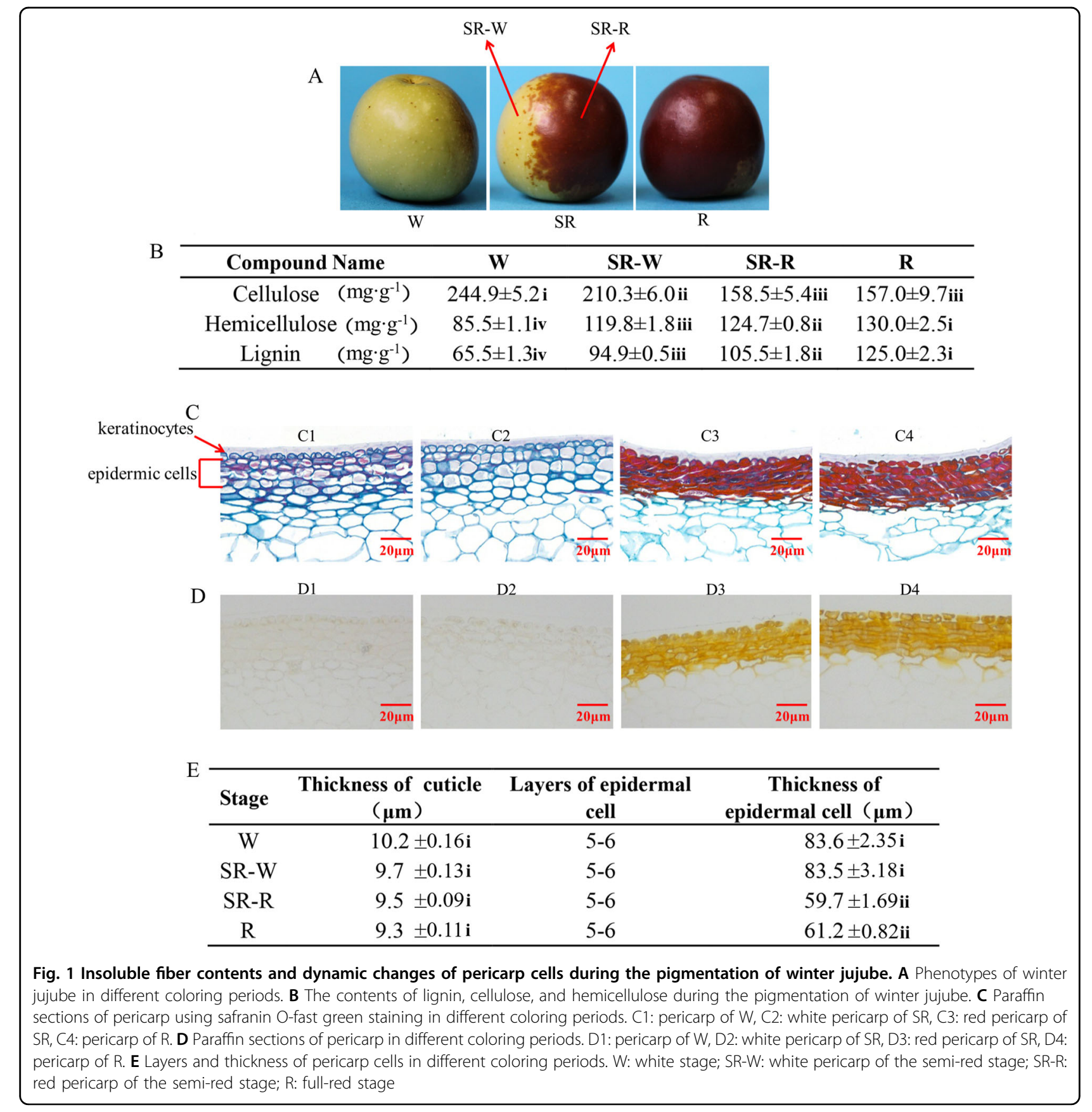

orange-red. The sections in Fig. 2A $(5 \mu \mathrm{m})$ and Fig. 2B $(10 \mu \mathrm{m})$ were relatively thin and transparent, making it hard to capture the structural details. The sections in Fig. 2E $(25 \mu \mathrm{m})$ and Fig. 2F $(30 \mu \mathrm{m})$ were relatively thick, turbid, and inflexible, making it difficult to observe cellular structures. Ultimately, sections at thicknesses of $15 \mu \mathrm{m}$ (Fig. 2C) and $20 \mu \mathrm{m}$ (Fig. 2D) illustrated the pericarp cell structure and pigment deposition relatively clearly and vividly. We found through the observations that the epidermal cells of the red jujube pericarp were orange-red in color, with a large amount of pigments deposited on the cell walls.

\section{Analysis of metabolites related to lignin biosynthesis in winter jujube pericarp during the pigmentation process}

The accumulation of 13 major metabolites related to lignin biosynthesis in winter jujube during the pigmentation process is illustrated in Fig. 3A. Phenylalanine and p-coumaric acid levels were decreased as pigmentation increased. In contrast, p-coumaroyl, sinapyl aldehyde, and 

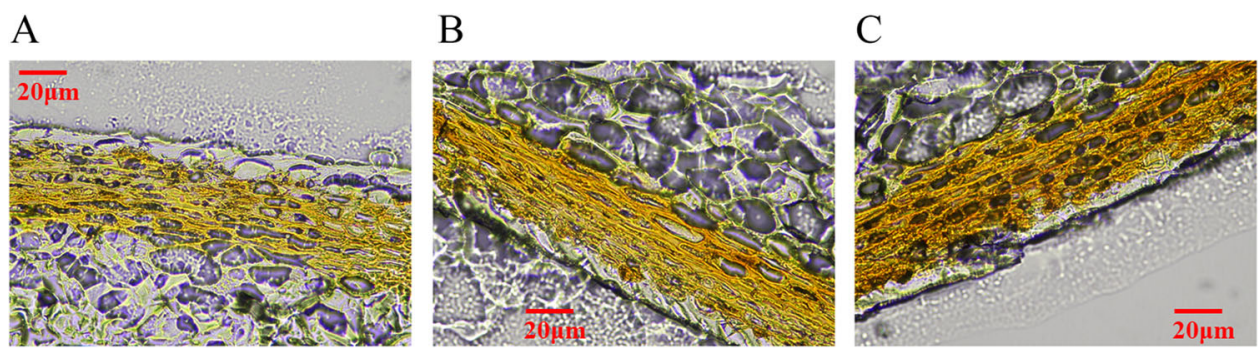

$\mathrm{D}$

$\mathrm{E}$
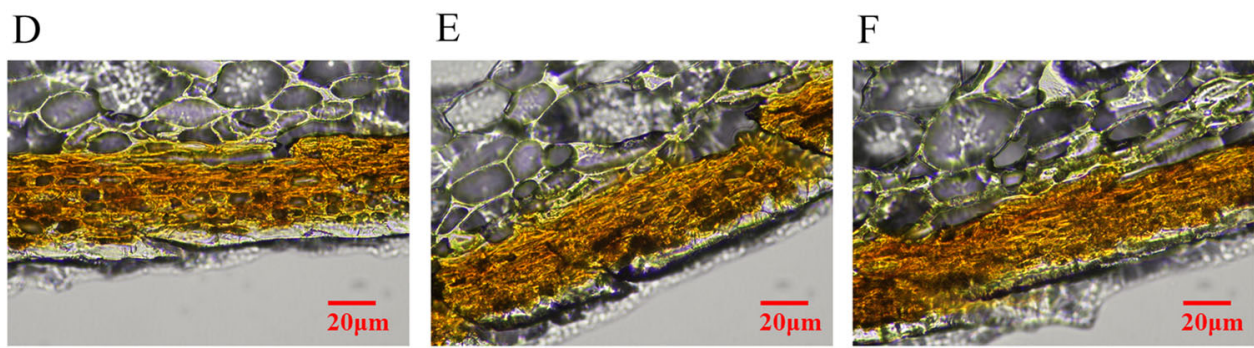

Fig. 2 Observation of pigment deposition sites in the pericarp of winter jujube using frozen sections. A Frozen section of winter jujube pericarp at the R stage with $5 \mu \mathrm{m}$ thicknesses. B Frozen section of winter jujube pericarp at the R stage with $10 \mu \mathrm{m}$ thicknesses. C Frozen section of winter jujube pericarp at the $\mathrm{R}$ stage with $15 \mu \mathrm{m}$ thicknesses. D Frozen section of winter jujube pericarp at the R stage with $20 \mu \mathrm{m}$ thicknesses. E Frozen section of winter jujube pericarp at the R stage with $25 \mu \mathrm{m}$ thicknesses. F Frozen section of winter jujube pericarp at the R stage with $30 \mu \mathrm{m}$ thicknesses

coniferyl aldehyde levels were higher in pigmented pericarps at the R and SR-R stages than in unpigmented pericarps at the W and SR-W stages. The contents of coniferyl alcohol and sinapyl alcohol first increased and then decreased, peaking at the SR-W stage. The p-coumaric alcohol content showed a declining trend, and its content was higher at the $\mathrm{W}$ stage than at the SR-W stage, with extremely low or no accumulation at the R and SR-R stages. These results indicate that G-S lignin rather than $\mathrm{H}$-lignin is the main monomer of winter jujube pericarp.

The gene expression levels of structural genes ( $P A L$, $C 4 H, 4 C L, C O M T, C C R, C A D, F 5 H$, and $P O D$ ) related to lignin biosynthesis in winter jujube were analyzed based on RNA-seq (Fig. 3B). Some genes were selected, and their expression levels were verified by qRT-PCR (Fig. S1). The results showed consistency between the transcriptome and qRT-PCR analysis. Specifically, the expression levels of $P A L$ and $C 4 H$ were downregulated during pericarp pigmentation. The expression levels of COMT genes (LOC107414981 and LOC107414981) were relatively high at the SR-R stage but low at the R stage. This pattern was different from the accumulating levels of sinapyl aldehyde, coniferyl aldehyde, and lignin. The expression levels of F5H (LOC107424406) and CCR (LOC107420974) were relatively high at the SR-R and R stages, consistent with the accumulation of sinapyl aldehyde, coniferyl aldehyde, and lignin. We deduced that F5H and CCR play key roles in G-S lignin biosynthesis. The expression of CAD (LOC107426959) was consistent with the accumulation of p-coumeric alcohol and may be the key gene for $\mathrm{H}$-lignin biosynthesis.

\section{Expression analysis of MYB TFs related to lignin biosynthesis}

A phylogenetic tree was constructed with MYB TFs in jujube, Arabidopsis, and poplar to screen out the potential MYB TFs that regulate lignin biosynthesis in winter jujube. As shown in Fig. 4A, the results showed that the winter jujube MYB TFs LOC107417581, LOC107425254, and LOC107418291 were on the same evolutionary branch as poplar PtrMYB3 and PtrMYB20 $0^{14}$ and Arabidopsis AtMYB46 $6^{15}$, AtMYB83 ${ }^{16}$, and AtMYB26 ${ }^{17}$ (Group A3). LOC107403729 was on a branch with Arabidopsis AtMYB58 and AtMYB63 ${ }^{18}$ (Group A2). LOC107434917 and LOC107414294 were on a branch with Arabidopsis AtMYB85 ${ }^{19}$ (Group A1). LOC107430169 and LOC107 430208 were on a branch with Arabidopsis AtMYB103 ${ }^{20}$ (Group A4). LOC107424134, LOC107434709, LOC10743 4532, and LOC107406469 were on a branch with Arabidopsis AtMYB75 ${ }^{19}$ (Group A7). LOC107432692 and $L O C 107415078$ were on a branch with poplar PtrMYB6 ${ }^{21}$ (Group A5). LOC107421590, LOC107415776, and LOC10 7404478 were on a branch with poplar PtrMYB93 ${ }^{22}$ (Group A6).

Seventeen jujube MYB TFs with potential regulation of lignin biosynthesis were screened out by phylogenetic analysis, among which nine TFs showed differential expression levels. The qRT-PCR results showed similar 

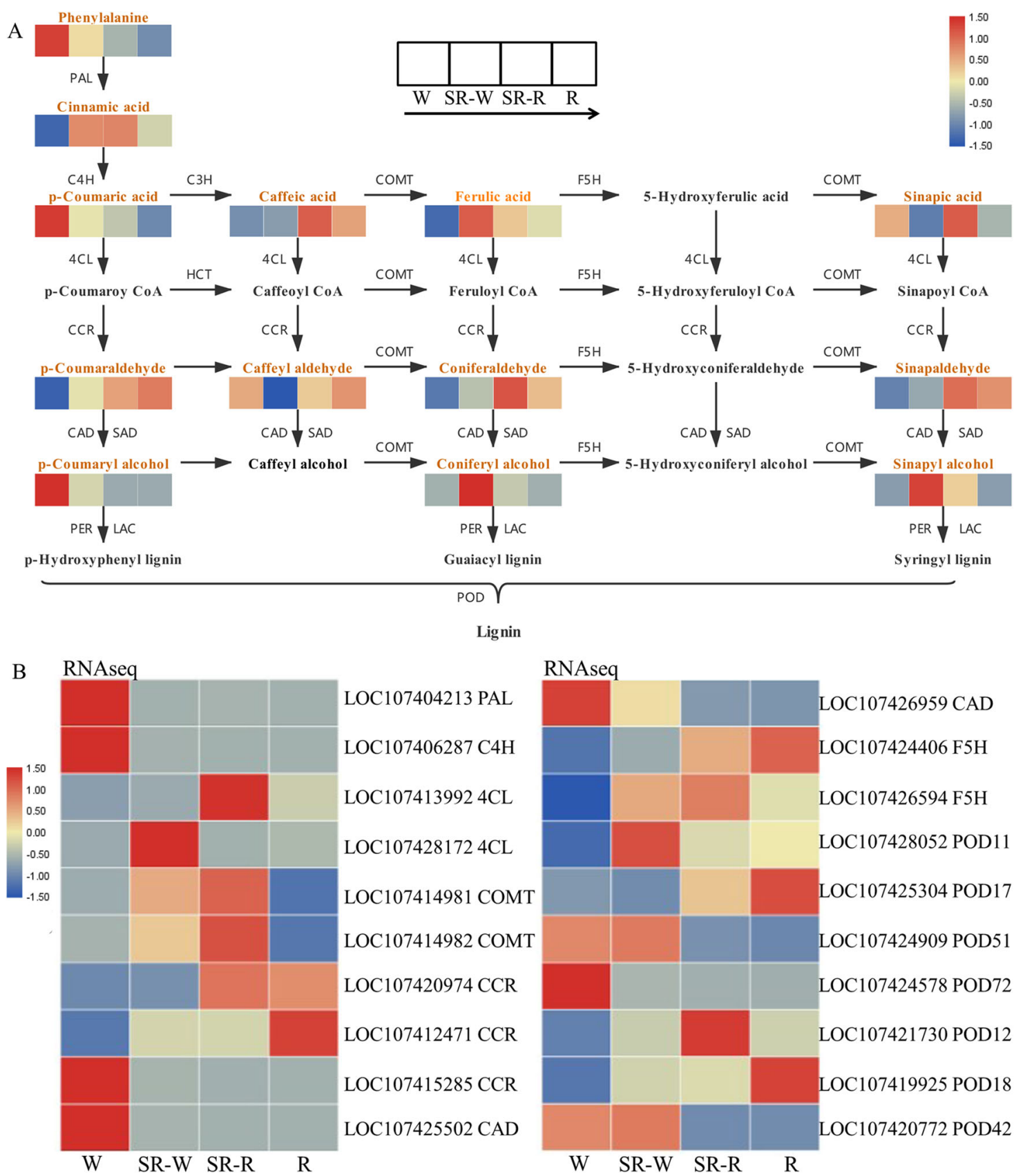

Fig. 3 Analyses of metabolites and structural genes related to the lignin biosynthesis pathway during the pigmentation of winter jujube pericarp. A Analyses of metabolites involved in lignin biosynthesis during the pigmentation of winter jujube pericarp. Colors from blue to red indicate that the abundance of metabolites is low to high, and a redder color indicates that the abundance of differentially abundant metabolites is high. B Heatmap analyses of structural genes involved in lignin biosynthesis during the pigmentation of winter jujube pericarp. Colors from blue to red indicate that the levels of gene expression are low to high, and a redder color indicates that the levels of gene expression are high. W white stage, SR-W white pericarp of the semi-red stage, SR-R red pericarp of the semi-red stage, R: full-red stage

expression trends as the transcriptome analysis (Fig. 5A). In particular, only the expression of LOC107425254 was in accordance with changes in the lignin content, and the expression levels of LOC107415078 and LOC107415776 decreased with increasing lignin content.

\section{Expression analysis of NAC TFs related to lignin biosynthesis}

To screen out the TFs associated with lignin biosynthesis in winter jujube from the NAC family, we used the amino acid sequences of 138 Arabidopsis NAC TFs ${ }^{23}, 289$ poplar 


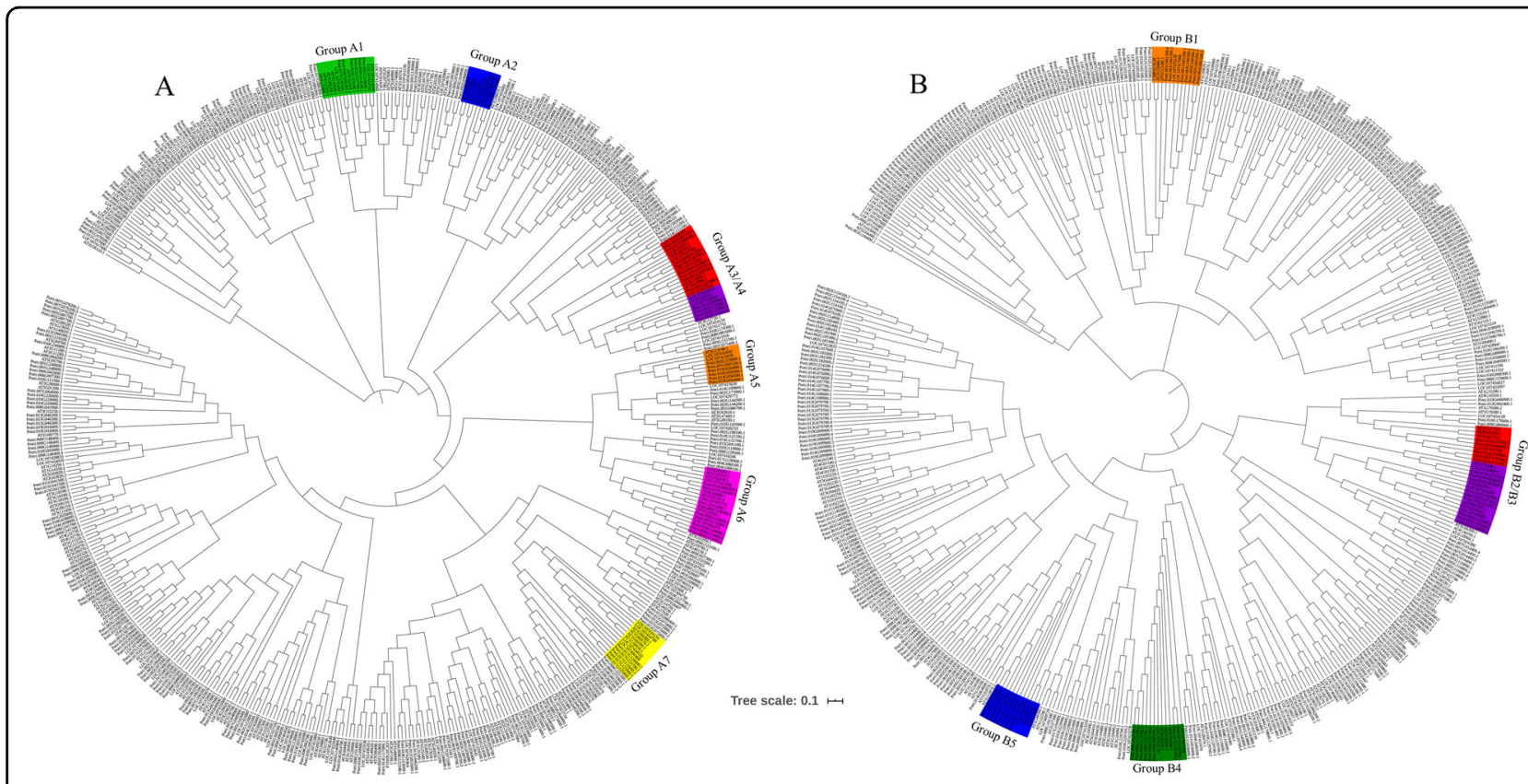

Fig. 4 Phylogenetic tree analyses of MYB and NAC TF families in Arabidopsis, poplar, and jujube. A Phylogenetic tree of 126 MYB TFs in Arabidopsis, 192 MYB TFs in poplar, and 99 MYB TFs in jujube. Accession numbers: AtMYB46 (AT5G12870), AtMYB83 (AT3G08500), AtMYB26 (AT3G13890), AtMYB58 (AT1G16490), AtMYB63 (AT1G79180), AtMYB75 (AT1G56650), AtMYB85 (AT4G22680), AtMYB103 (AT1G63910), PtrMYB3 (Potri.001G267300), PtrMYB20 (Potri.009G061500), PtrMYB6 (Potri.001G005100), and PtrMYB93 (Potri.004G138000.1). B Phylogenetic tree of 138 NAC TFs in Arabidopsis, 289 NAC TFs in poplar, and 101 NAC TFs in jujube. Accession numbers: AtNST1 (AT2G46770), AtNST2 (AT3G61910), AtNST3 (At1G32770), AtANAC012 (AT1G32770), AtANAC043 (AT2G46770), AtANAC066 (AT3G61910), AtANAC073 (AT4G28500), AtVNI1 (AT5G09330), AtVNI2 (AT5G13180), PtrWND1A (Potri.011G153300), PtrWND2A (Potri.014G104800), PtrWND1B (Potri.001G448400), and PtrWND2B (Potri.002G178700)

NAC TFs (http://planttfdb.cbi.pku.edu.cn), and 101 jujube NAC TFs (http://planttfdb.cbi.pku.edu.cn/) to construct a phylogenetic tree. As shown in Fig. 4B, Arabidopsis AtNST1 ${ }^{24}$, AtNST2, AtNST3, ANAC012, ANAC043, and $A N A C 066^{25}$ and poplar PtrWND1A/2A and PtrWND1B/ $2 B^{26}$ were located on the same branch, which was also shared by the jujube NAC TF LOC107423780 (Group B2/ B3). Arabidopsis AtANACO73 ${ }^{25}$ was on the same branch as jujube LOC107417198 and LOC107432797 (Group B4). Arabidopsis $A t V N I 2^{27}$ was on the same branch as jujube LOC107435239 and LOC107417668 (Group B1). Arabidopsis $\mathrm{AtVNI}^{27}$ and jujube $L O C 107425600$ were on the same branch (Group B5).

Six jujube NAC TFs associated with the regulation of lignin biosynthesis were screened out from the phylogenetic analysis. As shown in Fig. 5B, these six TFs were differentially expressed among samples and showed consistent expression levels between the transcriptome and qRT-PCR analysis, among which LOC107435239 and LOC107417668 exhibited relatively higher expression levels after jujube pigmentation, in line with the changes in lignin content.

\section{Ectopic expression in Arabidopsis and pericarp injection in winter jujube}

The above results identified three MYB (LOC107425254, LOC107415078, and LOC107415776) and two NAC
(LOC107435239 and LOC107417668) TFs as candidates associated with lignin biosynthesis. These candidate genes were ectopically expressed in Arabidopsis and injected into the winter jujube pericarp. The cross sections of Arabidopsis stems stained with safranin O-fast green are shown in Fig. 6A. Overexpression of MYB (LOC107425254) or NAC (LOC107435239) in Arabidopsis led to deep staining of the stem (Fig. 6A), which was associated with increased lignin levels (Fig. 6D). However, only light staining of stems was observed with MYB (LOC107415078) overexpression (Fig. 6A), indicating a limited lignin level (Fig. 6D).

The injected winter jujube pericarps are shown in Fig. 6B, and the pericarps stained with safranin $\mathrm{O}$-fast green are shown in Fig. 6C. The overexpression of MYB (LOC1074 25254) or NAC (LOC107435239) caused deep staining in the winter jujube pericarp around the injection sites, while overexpression of MYB (LOC107415078) resulted in only light staining. In addition, the lignin contents changed with the color of the staining (Fig. 6E). Further gene expression analysis showed that the overexpression of $M Y B$ (LOC107425254) in the winter jujube pericarp could significantly promote the expression of CCR (LOC107420974) and $F 5 H$ (LOC107424406), while overexpression of NAC ( $L O C 107435239$ ) could only increase the expression of $F 5 H$ (LOC107424406) (Fig. 6F). In contrast, the overexpression of $M Y B$ (LOC107415078) in the winter jujube pericarp 


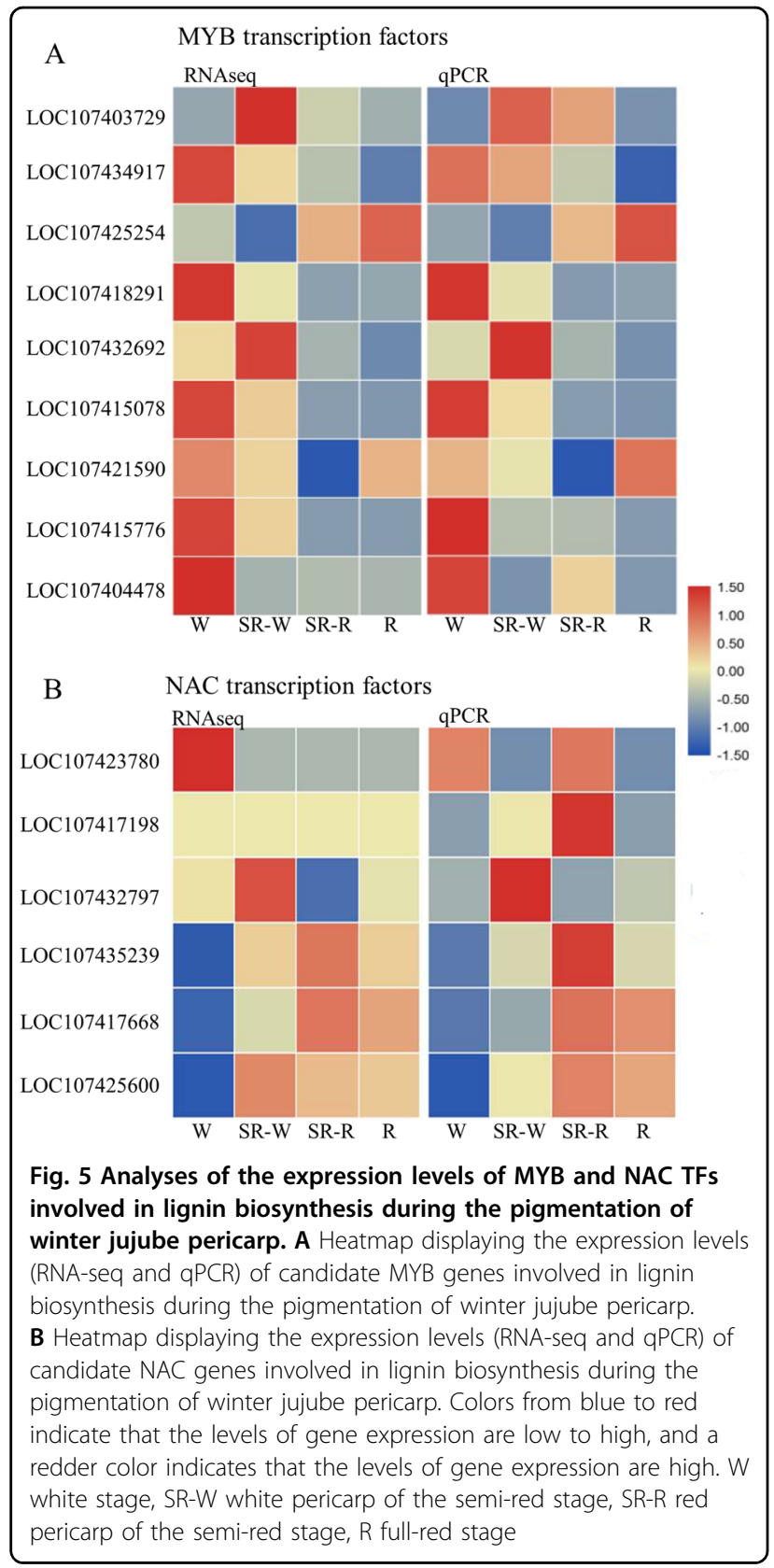

reduced the expression of $C C R$ (LOC107420974) and F5H (LOC107424406) (Fig. 6F).

\section{Discussion}

\section{Changes in cell structure, cell wall lignification, and} pigment deposition during the pigmentation process of winter jujube pericarp

Jujube pericarp pigmentation develops with the ripening process of fruits, which is very complex and involves many physiological processes, including cell senescence, pigment deposition, and metabolite accumulation. Cell senescence includes changes and modifications of cell wall components, as well as aging and death of organs ${ }^{28}$. Similarly, the cell structure of the jujube pericarp also changes with the pigmentation process, which has rarely been reported. Studies on the cell structure of jujube pericarp have mainly focused on the changes in cell structure during fruit cracking and fruit development ${ }^{29}$ rather than ripening and pigmentation. This study revealed that jujube pericarp cells are mainly composed of cuticle cells and epidermal cells (Fig. 1C, D). Moreover, the shape, size, and arrangement of epidermal cells varied with the ripening and pigmentation processes of the fruit. Specifically, the cell shape changed from oblate to oblong, and the arrangement changed from compact to relatively loose during the pigmentation process (Fig. 1C, D), which is consistent with the results of the previous research ${ }^{4}$. Moreover, it has been reported that the epidermal cell thickness and layer number of jujube fruits decrease with fruit ripening. In contrast, our study confirmed the decrease in epidermal cell thickness with fruit ripening, but the layer number remained unchanged (Fig. 1E). This discrepancy between the two studies may be associated with the difference in the jujube varieties used.

The lignification of the jujube pericarp is due to lignin deposition in the cell wall during cell development. Studies have shown organelle degradation, vacuole rupture, and cell content disappearance after cell wall lignification, leading to programmed cell death ${ }^{30}$. Zhao ${ }^{31}$ found that the contents of cellulose and hemicellulose in the pericarp decreased with fruit ripening. $\mathrm{Li}^{32}$ showed that the lignin biosynthetic pathway was activated at the transcriptional level during the coloration of green cotton fibers, indicating the function of lignin in pigmentation. In this study, our results showed a decrease in the cellulose content and an increase in the hemicellulose and lignin contents (Fig. 1B). The paraffin sections of jujube pericarp stained with safranin $\mathrm{O}$-fast green revealed the lignification of pigmented epidermal cells reflected by the red color (Fig. 1C). In addition, frozen sections of jujube pericarp at the $\mathrm{R}$ stage showed that the epidermal cells were full of chromoplasts, with deposition of a large amount of pigments on the cell wall (Fig. 2). This phenomenon might be caused by the release of pigments (e.g., anthocyanins) from the cell vacuoles during cell wall lignification and cell apoptosis. Furthermore, the orange-red color of thickened cell walls might be associated with the high content of lignin or the polymerization of lignin with the spilled pigments into macromolecular phenols, which needs further verification.

\section{Analysis of lignin biosynthesis and structural gene expression during the pigmentation process of winter jujube pericarp}

It has been shown that the pericarp cells of Chinese jujube change with cell lignification, which is closely 


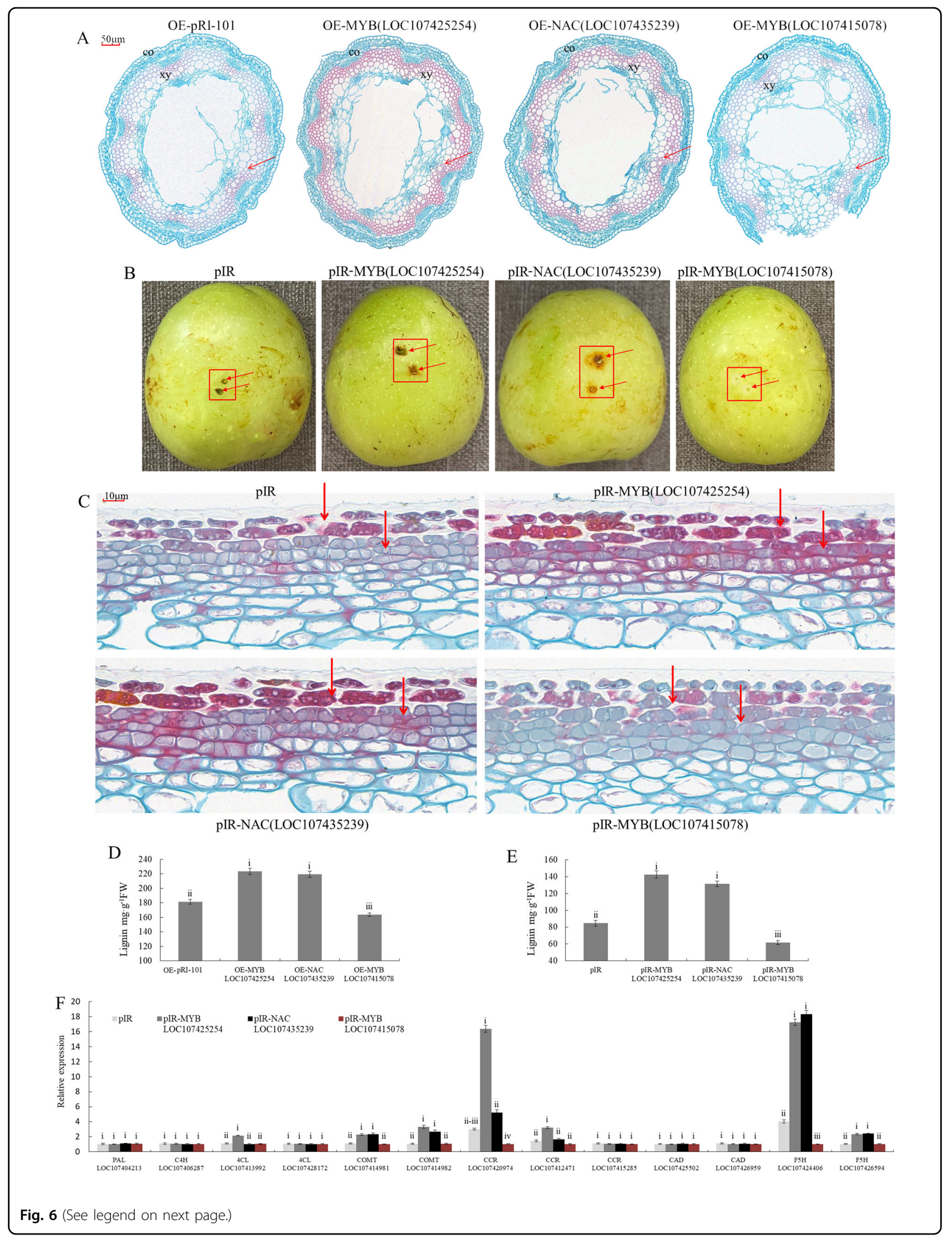


(see figure on previous page)

Fig. 6 The function of MYB and NAC candidate transcription factors involved in lignin biosynthesis. Significance tests are shown as i, ii, iii, and iv. Different letters above the columns denote significant differences $(P<0.01)$. A Cross sections of stems stained with safranin O-fast green in transgenic Arabidopsis. OE-pRI-101: overexpressing empty vectors. OE-MYB (LOC107425254)/(LOC107415078): overexpression of MYB (LOC107425254) or MYB (LOC107415078) in Arabidopsis, OE-NAC (LOC107435239): overexpression of NAC (LOC107435239) in Arabidopsis. B The winter jujube pericarp was injected with the mixed vectors and stored in a phytotron at $25^{\circ} \mathrm{C}$ for 3 days. pIR; IL60-1 + IL60-2. pIR-MYB (LOC107425254): IL60-1 + MYB (LOC107425254)-IL60-2. pIR-MYB (LOC107415078): IL60-1 + MYB (LOC107415078)-IL60-2. pIR-NAC (LOC107415078): IL60-1 + NAC (LOC107435239)-IL60-2. C The injected winter jujube pericarp stained with safranin O-fast green. D Lignin contents of Arabidopsis stem. E Lignin contents in the winter jujube pericarp around the injection sites. $\mathbf{F}$ The expression of structural genes associated with lignin biosynthesis in the winter jujube pericarp around the injection sites

related to the accumulation of $\operatorname{lignin}^{33}$. Lignin consists of three monomers: H-lignin, G-lignin, and S-lignin. Generally, gymnosperms primarily have G-lignin, monocotyledons primarily have G-S-H lignin, and dicotyledons primarily have G-S lignin ${ }^{34}$. We studied the accumulation of 13 intermediate metabolites of the lignin biosynthesis pathway through metabolomics analysis, and the results showed that p-coumaric alcohol, the precursor of $\mathrm{H}$-lignin, did not accumulate in the pericarp of winter jujube at the SR and R stages. Moreover, coniferyl alcohol, the precursor of G-lignin, and sinapyl alcohol, the precursor of S-lignin, were first increased and then decreased, with a peak at the SR stage (Fig. 3A), indicating the dominance of G-S lignin in the pericarp of winter jujube.

There are eight main structural genes involved in the lignin biosynthesis pathway ${ }^{35}$, among which $P A L, C 4 H$, and $4 C L$ are the pro-structural genes for lignin biosynthesis. COMT and F5H are the key genes for G-S lignin biosynthesis. In alfalfa, downregulation of COMT expression reduced the content of G-lignin, while S-lignin remained unchanged $^{36}$. Takeda et $a l^{37}$ overexpressed $F 5 H$ in rice to increase the S-lignin content. Moreover, overexpression of $B p C C R 1$ in birch increased lignin content by $14.6 \%$, while knockdown of this gene lowered the lignin content by $6.3 \%^{38}$. In our study, the expression levels of $C O M T$ genes (LOC107414981 and LOC107414981) were relatively high at the SR_R stage but low at the R stage, which was not consistent with the accumulation of coniferyl aldehyde, sinapyl aldehyde, and lignin (Fig. 3 and Fig. S1). The expression levels of F5H (LOC107424406) and CCR (LOC107420974) were relatively high at the SR_R and R stages, which was consistent with the accumulation of coniferyl aldehyde, sinapyl aldehyde, and lignin (Fig. 3 and Fig. S1). The results indicated that F5H (LOC107424406) and CCR (LOC107420974) might be the key genes for lignin biosynthesis in the pericarp of winter jujube. Notably, we also found that the expression of CAD (LOC107426959) was consistent with the accumulation of p-coumaric alcohol (Fig. 3), which was in line with a previous report that $C A D$ was one of the specific enzymes in the lignin biosynthesis pathway, and the loss of CAD activity impacted lignin composition more than lignin content ${ }^{39}$.

\section{Regulation of TFs related to lignin during the pigmentation process of winter jujube pericarp}

MYB TFs are important regulators of lignin metabolism. Some MYB TFs participate in the regulation of the formation and lignification of secondary cell walls. For example, overexpression of AtMYB46 and AtMYB83 activated related structural genes in the lignin biosynthesis pathway, resulting in lignin accumulation and secondary cell wall thickening in Arabidopsis ${ }^{15,16}$. In this study, we revealed that the MYB TF LOC107425254, which was on the same branch as AtMYB46 and AtMYB83 (Fig. 4A), was upregulated with increasing lignin content in the winter jujube pericarp (Fig. 5A), indicating positive regulation of the lignification of winter jujube pericarp cells. Increased lignin content was detected in Arabidopsis or winter jujube pericarp overexpressing MYB (LOC107425254) (Fig. 6A-E). Further analysis showed that it could significantly promote the expression of CCR (LOC107420974) and F5H (LOC107 424406) (Fig. 6F), suggesting that MYB (LOC107425254) positively controls lignin content by regulating $C C R$ (LOC107420974) and F5H (LOC107424406).

PtrMYB6 negatively regulated lignin biosynthesis in poplars. Specifically, reduced lignin content and the number of cell layers, as well as thinner cell walls, were observed in the transgenic plants overexpressing PtrMYB6 ${ }^{21}$. Another study also showed that poplars overexpressing PtrMYB93 had lower lignin contents. Alternatively, when PtrMYB93 was knocked out, the expression levels of key structural genes in the lignin biosynthesis pathway were significantly upregulated, and the lignin content was increased ${ }^{22}$. In this study, we found that the levels of the MYB TFs LOC107415776 and LOC107415078 were downregulated with increasing lignin content in the winter jujube pericarp (Fig. 5A). Phylogenetic analysis showed that LOC107415776 and PtrMYB6 were on the same branch (Fig. 4A), while LOC107415078 and PtrMYB93 were on the same branch (Fig. 5A), indicating that they negatively regulate lignin biosynthesis. However, in this study, only MYB (LOC107415078) was predicted to negatively regulate lignin biosynthesis by reducing the expression of $C C R$ (LOC107420974) and F5H (LOC107424406) (Fig. 6). 


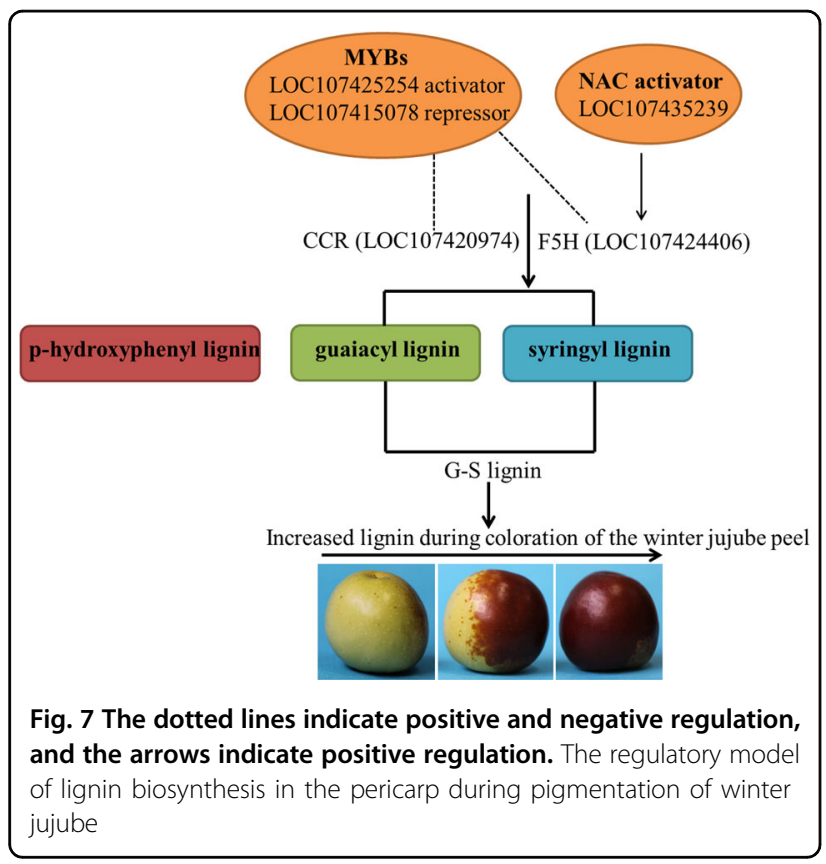

NAC TFs, such as AtNST1/2/3 $3^{24,25}$ and AtVNI1/2 $2^{27}$ of Arabidopsis and PtrWND1A/2A and PtrWND1B/2B of poplar $^{26}$, also play an important role in lignin biosynthesis. In this study, two NAC TFs, LOC107435239, and LOC107417668 were selected for analysis, and their expression levels were upregulated with increasing lignin content (Fig. 5B). Phylogenetic analysis indicated that they were on the same branch as AtVNI2 (Fig. 4B). AtVNI2 can inhibit the activation of the AtVDN7 TF, which specifically regulates the development of secondary cell wall ${ }^{27}$. Therefore, we speculated that LOC107435239 and LOC107417668 might be involved in the metabolic process of secondary cell wall development. Only the overexpression of NAC (LOC107435239) increased lignin content in Arabidopsis mutants (Fig. 6A, D). Further analysis also showed that the overexpression of $N A C$ (LOC107435239) positively regulated lignin biosynthesis in the winter jujube pericarp around the injection sites (Fig. 6B-E) and significantly promoted the expression of F5H (LOC107424406) (Fig. 6F).

\section{Conclusion}

A regulatory model of lignin biosynthesis in winter jujube pericarp during pigmentation is proposed based on the results from this study (Fig. 7). With the increase in lignin content and the lignification of cell walls during pigmentation of winter jujube pericarp, the thickness of the epidermal cells decreased. The lignin of the winter jujube pericarp was mainly G-S lignin, and $F 5 H$ (LOC107424406) and CCR (LOC107420974) were preliminarily identified as the key genes for G-S lignin biosynthesis. This study also identified an MYB activator
(LOC107425254) and an MYB repressor (LOC107415078) associated with lignin biosynthesis by regulating $C C R$ (LOC107420974) and F5H (LOC107424406), while the NAC (LOC107435239) TF significantly promoted the expression of $F 5 H$ (LOC107424406) and positively regulated lignin biosynthesis. In conclusion, these results revealed the metabolic pathway and key genes controlling lignin biosynthesis during pigmentation of winter jujube pericarp and provide a basis for further research on lignin regulation.

\section{Materials and methods Plant materials}

Winter jujube fruits were collected from the germplasm repository of Shandong Institute of Pomology in 2018. The repository was located in the Taishan District of Tai'an City, Shandong Province, China. The growth conditions were a semihumid continental monsoon climate zone, a plain region with clay loam soils, with an average annual temperature of $13{ }^{\circ} \mathrm{C}$, average annual precipitation of $697 \mathrm{~mm}$, and a frost-free period of 195 days. Three 13-year-old healthy jujube plants with similar growth statuses and under unified management were selected for the study. Briefly, ten jujube fruits with homogeneous size, shape, and color were randomly collected from different locations of the sample trees at the white stage (W), semi-red stage (SR), and full-red stage (R). Fruit samples with infection of diseases and pests or mechanical damage were strictly excluded from the study. Different samples were mixed as a biological replicate and used in triplicate for each experiment. After collection, samples were immediately brought back to the laboratory, washed, peeled, frozen in liquid nitrogen, and stored at $-80^{\circ} \mathrm{C}$ for subsequent uses.

\section{Determination of insoluble fiber}

The lignin content was determined according to the method of Shilton ${ }^{40}$ with slight modification. Briefly, $0.5 \mathrm{~g}$ of sample was homogenized with $95 \%$ ethanol and centrifuged at $4000 \mathrm{rpm}$ for $10 \mathrm{~min}$. The pellet was washed three times with $95 \%$ ethanol and then rinsed three times with ethanol: $n$-hexane (1:1) solution, and the precipitate was collected and dried. The dry mass was placed in the test tube, dissolved in $0.5 \mathrm{~mL}$ of $25 \%$ bromoacetyl, and incubated at $70{ }^{\circ} \mathrm{C}$ in a water bath for $30 \mathrm{~min}$, followed by the addition of $0.9 \mathrm{~mL}$ of $2 \mathrm{~mol} \cdot \mathrm{L}^{-1} \mathrm{NaOH}$ to terminate the reaction. Subsequently, $5 \mathrm{~mL}$ of glacial acetic acid and $0.1 \mathrm{~mL}$ of $7.5 \mathrm{~mol} \cdot \mathrm{L}^{-1}$ hydroxylamine hydrochloride were added. The mixture was centrifuged for $5 \mathrm{~min}, 0.1 \mathrm{~mL}$ of supernatant was taken and diluted by adding $3.0 \mathrm{~mL}$ of glacial acetic acid, and the absorbance value at $280 \mathrm{~nm}$ was determined.

The cellulose content was measured by the Cellulase Assay Kit according to the manufacturer's instructions. 
Specifically, $0.3 \mathrm{~g}$ of sample was first incubated in $80 \%$ ethanol for $20 \mathrm{~min}$ at $95^{\circ} \mathrm{C}$. After cooling, the sample was centrifuged at $4000 \mathrm{rpm}$ for $10 \mathrm{~min}$. The pellets were vortexed with $1.5 \mathrm{ml}$ of $80 \%$ ethanol for $2 \mathrm{~min}$, followed by 2 min of centrifugation at $4000 \mathrm{rpm}$. After removal of ethanol, the sample was washed with acetone, followed by incubation with $1 \mathrm{~mL}$ of reagent I for $15 \mathrm{~h}$. Samples were centrifuged, and the pellets were dried in an oven at $105^{\circ} \mathrm{C}$ to constant weights. Five milligrams of dried sample was homogenized using $0.5 \mathrm{~mL}$ of deionized water and incubated on ice. Slowly, $0.75 \mathrm{~mL}$ of concentrated sulfuric acid was added, and the samples were incubated for another $30 \mathrm{~min}$. The supernatant after centrifugation at $4000 \mathrm{rpm}$ for $10 \mathrm{~min}$ was taken and diluted 20 times using deionized water for subsequent measurement. For the preparation of the working solution, $4 \mathrm{~mL}$ of reagent III was added into reagent II. Seventy microliters of working solution and $630 \mu \mathrm{L}$ of concentrated sulfuric acid were added to $300 \mu \mathrm{L}$ of the prepared sample solution and deionized water, respectively, and incubated at $95^{\circ} \mathrm{C}$ for $10 \mathrm{~min}$, and the absorbance at $620 \mathrm{~nm}$ was recorded after cooling.

The hemicellulose content was determined by using a commercial hemicellulose detection kit. According to the manufacturer's instructions, $2 \mathrm{~mL}$ of reagent I was added to $0.1 \mathrm{~g}$ of the sample, incubated at $90^{\circ} \mathrm{C}$ for $10 \mathrm{~min}$, and centrifuged at $5000 \mathrm{rpm}$ for $10 \mathrm{~min}$. The pellets were washed three times with $1 \mathrm{~mL}$ of deionized water and kept in an oven at $105^{\circ} \mathrm{C}$ until they reached a constant weight, followed by the addition of $1 \mathrm{~mL}$ of reagent II. The mixture was incubated at $90^{\circ} \mathrm{C}$ for $1 \mathrm{~h}$, followed by the addition of $0.1 \mathrm{~mL}$ of reagent III and $1 \mathrm{~mL}$ of reagent IV and mixing until the color was reddish. The solution was centrifuged at $5000 \mathrm{rpm}$ for $10 \mathrm{~min}$, and $0.2 \mathrm{~mL}$ of the supernatant was thoroughly mixed with $0.15 \mathrm{~mL}$ of reagent $\mathrm{V}$ and $0.65 \mathrm{~mL}$ of deionized water. After $5 \mathrm{~min}$ of incubation in a water bath at $90^{\circ} \mathrm{C}$, absorbance at $540 \mathrm{~nm}$ was recorded.

\section{Preparation and observation of paraffin sections}

Pericarp samples with a thickness less than $0.2 \mathrm{~cm}$ were sliced from the winter jujube fruits from the W stage, SRW stage, SR-R stage, and R stage cut into $0.3 \mathrm{~cm} \times 0.3 \mathrm{~cm}$ pieces, fixed with FAA fixing solution, and incubated in $70 \%$ ethanol solution for $12 \mathrm{~h}$. Furthermore, the pericarp samples were dehydrated consecutively in $80 \%$ ethanol for $60 \mathrm{~min}, 90 \%$ ethanol for $30 \mathrm{~min}$, and $100 \%$ ethanol for $15 \mathrm{~min}$. The dehydrated samples were further incubated sequentially in anhydrous ethanol/xylene solution for $15 \mathrm{~min}$, in xylene solution for $3 \mathrm{~min}$, and in paraffin/ xylene for $30 \mathrm{~min}$, followed by paraffin embedding for $80 \mathrm{~min}$. The embedded samples were sliced into sections with a thickness of $5 \mu \mathrm{m}$. The paraffin slices were picked out with toothpicks and placed on slides, developed with warm water at $35^{\circ} \mathrm{C}$, placed on a slide coated with a thin layer of protein glycerin, and dried in an oven at $38^{\circ} \mathrm{C}$.

The samples at the same stage were then treated with safranin O-fast green staining as follows: the sections were placed in xylene I for $20 \mathrm{~min}$, xylene II for $20 \mathrm{~min}$, anhydrous ethanol I for $5 \mathrm{~min}$, anhydrous ethanol II for $5 \mathrm{~min}$, and $75 \%$ ethanol for $5 \mathrm{~min}$, and then rinsed with ultrapure water. The treated samples were stained in $0.1 \%$ safranin solution for $2 \mathrm{~h}$, rinsed with ultrapure water, and placed in 50,70 , and $80 \%$ ethanol for $5 \mathrm{~s}$. The samples were then stained with $0.15 \%$ solid green dye for $60 \mathrm{~s}$, rinsed with ultrapure water and glacial acetic acid solution, dehydrated with $95 \%$ ethanol for $10 \mathrm{~s}$, dehydrated with anhydrous ethanol for $10 \mathrm{~s}$, processed in xylene for $5 \mathrm{~min}$, and sealed with neutral glue. Nonstained and stained slices were observed under an optical microscope (Olympus BX53) and photographed. Changes in the epidermal cell layer and cell structure of the jujube pericarp were observed. The thicknesses of cuticle and epidermal cells were measured with CaseViewer software (The Digital Pathology Company).

\section{Preparation and observation of frozen sections}

Fresh winter jujube fruits at the $\mathrm{R}$ stage were cut into $0.5 \mathrm{~cm} \times 0.5 \mathrm{~cm} \times 0.2 \mathrm{~cm}$ pieces, placed in the sample disc, and surrounded by OCT embedding drops. The sample disc was slowly placed into the liquid nitrogen tank. When the disc bottom reached the liquid nitrogen, the disc was held for 10-20 s before being immersed in liquid nitrogen. The frozen samples were sliced into sections with thicknesses of $5,10,15,20,25$, and $30 \mu \mathrm{m}$. The sections were observed and photographed under an optical microscope (Olympus BX53). The number of epidermal cell layers, the change in cell structures, and pigment deposition were recorded.

\section{Ultraperformance liquid chromatography-mass spectrometry (UPLC-MS) analysis}

Approximately $0.1 \mathrm{~g}$ of winter jujube pericarp sample was ground into powder in liquid nitrogen, and $500 \mu \mathrm{L}$ of $80 \%$ methanol solution containing $0.1 \%$ formic acid was added; then, the sample was vortexed and placed in an ice bath for $5 \mathrm{~min}$. After centrifugation at $15,000 \mathrm{rpm}$ for $10 \mathrm{~min}$ at $4{ }^{\circ} \mathrm{C}$, the sample was diluted with a $53 \%$ methanol solution and centrifuged at $15,000 \mathrm{rpm}$ for $20 \mathrm{~min}$ at $4{ }^{\circ} \mathrm{C}$. The supernatant was used for UPLC-MS analysis. Aliquots from each test sample were mixed as quality control (QC) samples.

The UPLC assay was performed on a Shim-pack Shimadzu CBM30A equipped with a Waters Acquity UPLC HSS T3 C18 column of $1.8 \mu \mathrm{m}(2.1 \mathrm{~mm} \times 100 \mathrm{~mm})$. The experimental conditions were as follows. flow rate: $0.4 \mathrm{ml}$ $\min ^{-1}$; column temperature: $40^{\circ} \mathrm{C}$; injection volume: $2 \mu \mathrm{L}$; mobile phase A: $0.04 \%$ acetic acid aqueous solution, 
mobile phase B: $0.04 \%$ acetonitrile solution. Sample elution was established by mixing mobile phase $\mathrm{A} /$ mobile phase B (V/V) in a linear gradient: 0 min was 95:5, $11.0 \mathrm{~min}$ was $5: 95,12.0 \mathrm{~min}$ was $5: 95,12.1 \mathrm{~min}$ was $95: 5$, and $15.0 \mathrm{~min}$ was $95: 5$.

Mass spectrum analysis was carried out by a triple fourpole tandem mass spectrometer (Applied Biosystems 6500 QTRAP) with the following parameters: temperature $500^{\circ} \mathrm{C}$, voltage $5500 \mathrm{~V}$, curtain gas (CUR) $25 \mathrm{psi}$, and collision-activated dissociation (CAD) as high.

\section{Qualitative and quantitative analysis of metabolites and data analysis}

Based on the Metware Database (MWDB) and other public metabolite information databases, metabolite characterization was carried out according to the secondary spectral information. During the analysis, isotope signals, repeated $\mathrm{K}^{+}, \mathrm{Na}^{+}$, and $\mathrm{NH}_{4}^{+}$ion signals, and repetitive signals of fragment ions contained in other larger molecules were removed. Metabolites were quantitatively analyzed by multiple reaction monitoring (MRM) using Triple Quad mass spectrometry.

The metabolite data were analyzed using Analyst 1.6.1 software (AB Sciex, Ontario, Canada). Principal component analysis (PCA) was used to analyze the differences in population metabolism among samples and the degree of variation among samples within a group. The metabolites were analyzed by partial least square discriminant analysis (OPLS-DA), and the metabolites with variable importance in projection (VIP) $\geq 1$ and $P$ value $\leq 0.5$ were identified as differentially abundant metabolites. Hierarchical clustering analysis (HCA) and metabolite correlation analysis were used to reveal the relationship between metabolites and samples.

\section{Transcriptome analysis}

Total RNA was extracted from the pericarp of winter jujube using an RNAprep Pure Polysaccharide polyphenol total plant RNA extraction kit (TransGen Biotech, Beijing, China). The qualified total RNA was used for subsequent library construction and sequencing by the Illumina NovaSeq 6000 platform.

The reference genome sequence and functional gene annotation files were retrieved from https://ftp.ncbi.nlm. nih.gov/genomes/all/GCF/000/826/755/GCF_00082675

5.1_ZizJuj_1.1. Hisat2 V2.0.5 was used to compare clean paired-end reads with the reference genome. Reads of each gene in each sample were calculated using featureCounts V1.5.0-p3, and the value of fragments per kilobase of exon per million mapped reads (FPKM) of each gene was calculated based on the length of the gene.

DESeq2 R software (version 1.16.1) was used to compare expression differences between paired groups. Genes with $P<0.05$ and $\mid \log 2$ (fold change) $\mid>0$ after correction were considered significantly differentially expressed genes for subsequent analysis.

Gene Ontology (GO) enrichment and KEGG enrichment analysis of differentially expressed genes were achieved by clusterProfiler $\mathrm{R}$ software, in which the gene length bias was corrected. GO terms and KEGG terms with adjusted $P<0.05$ were used as significantly enriched functional terms for subsequent analysis.

\section{Quantitative real-time polymerase chain reaction (qRT- PCR)}

The selected genes and TFs were verified by qRT-PCR. Specific primers were designed using Primer 6.0 according to the whole genome sequences of winter jujube. The TransStart $^{\circledR}$ Top Green qPCR SuperMix kit was used for detection. Each sample was analyzed in triplicate, with ZjActin used as the internal reference gene. The $\mathrm{Ct}$ values were read under default conditions, and the $2^{-\Delta \Delta C T}$ method was used for data analysis ${ }^{41}$.

\section{Ectopic expression in Arabidopsis and pericarp injection of winter jujube}

Ectopic expression was carried out as described by Wang et al. ${ }^{42}$, with slight alterations. The recombinant plasmids containing candidate genes were transformed into Agrobacterium tumefaciens GV3101, which was utilized to infect Arabidopsis. The T1 transgenic Arabidopsis plants were then selected by plating onto MS medium containing hygromycin. The hygromycin-resistant seedlings were moved to soil and grown in a growth chamber (NingboJiangnan, http://www.nbjnyq.com/). The T2 seeds were then collected and grown as described above, and the lignin contents of their stems were determined.

Pericarp injection assays were carried out as described previously ${ }^{43}$. The overexpression of viral vectors MYB (LOC107425254)-IL60-2, MYB (LOC107415078)-IL60-2, and NAC (LOC107435239)-IL60-2 was generated by inserting the corresponding CDSs into the IL60-2 vector. The IL60-1 vector was used as an auxiliary plasmid. The mixed vectors (IL60-1: IL60-2=1: 1; $500 \mu \mathrm{L}: 100 \mu \mathrm{M}$ acetosyringone, $10 \mathrm{mM}$ 2-morpholinoethanesulfonic acid) were injected into the fruit pericarp using a $1 \mathrm{~mL}$ medical syringe. Then, the fruits were stored in a phytotron at $25^{\circ} \mathrm{C}$ for 3 days.

\section{Statistical analysis}

All results shown represent the mean of three independent assays. Significant differences between groups were determined by Duncan's new multiple range test, with significance tests denoted as i, ii, iii, and iv. Different lowercase letters in figures and tables denote significant differences $(P<0.05)$. 


\section{Acknowledgements}

The authors would like to thank TopEdit (www.topeditsci.com) for its linguistic assistance during the preparation of this manuscript. This work was supported by the National Key R\&D Program of China (2019YFD1001605), the Agricultural Science and Technology Innovation Project of Shandong Academy of Agricultural Sciences (CXGC2017D01 and CXGC2018E22), Shandong Academy of Agricultural Sciences introduction and training of high-level talents (1-18078), the Science and Technology Research Project of University in Hebei Province (QN2020205), the Shandong Key Research and Development Program (2016GNC113015 and 2019GNC106061), the Shandong Agricultural Applied Technology Innovation Project (2017), and the Shandong Provincial Natural Science Fund (ZR2020QC141).

\section{Author details}

'Shandong Institute of Pomology, Tai'an, Shandong 271000, China. ${ }^{2}$ School of Landscape and Ecological Engineering, Hebei University of Engineering, Handan, Hebei 056038, China. ${ }^{3}$ College of Food Science and Engineering, Shandong Agricultural University, 61 Daizong Street, Tai'an 271018 Shandong, P.R. China. ${ }^{4}$ Research Center of Chinese Jujube, Hebei Agricultural University, Baoding, Hebei 071000, China

\section{Data availability}

Supporting data not included in the manuscript are stored in (https://pan. baidu.com/share/init?surl=G_N60yxSOw-b4zKyHsNMgg, password: 2021).

\section{Competing interests}

The authors declare no competing interests.

Supplementary information The online version contains supplementary material available at https://doi.org/10.1038/s41438-021-00670-4.

Received: 2 December 2020 Accepted: 30 July 2021

Published online: 01 November 2021

\section{References}

1. Liu, M. J., Wang M. Germplasm Resources of Winter jujube [M] (China Forestry Publishing House, 2009).

2. Tahergorabi, Z. et al. "Ziziphus jujuba": a red fruit with promising anticancer activities. Pharmacogn. Rev. 9, 99-106 (2015).

3. Cheng, D., Cao, J. \& Jiang, W. The protective effects of polyphenols from jujube peel (Ziziphus Jujuba Mill) on isoproterenol-induced myocardial ischemia and aluminum-induced oxidative damage in rats. Food Chem. Toxicol. 50, 1302-1308 (2012).

4. Boerjan, W., Ralph, J. \& Baucher, M. Lignin biosynthesis. Annu Rev. Plant Biol. 54, 519-546 (2003).

5. Korth, K. L. et al. Changes in phenylpropanoid metabolites associated with somatic reversion of homology-dependent silencing of phenylalanine ammonia-lyase in tobacco. Physiol. Plant. 111, 137-143 (2008).

6. Schilmiller, A. et al. Mutations in the cinnamate 4-hydroxylase gene impact metabolism, growth and development in Arabidopsis. Plant J. 60, 771-782 (2009).

7. Besseau, S. et al. Flavonoid accumulation in Arabidopsis repressed in lignin synthesis affects auxin transport and plant growth. Plant Cell 19, 148-162 (2007).

8. Thbenin, J. et al. The simultaneous repression of CCR and CAD, two enzymes of the lignin biosynthetic pathway, results in sterility and dwarfism in Arabidopsis thaliana. Mol. Plant 4, 70-82 (2011).

9. Zhu, L. et al. CmMYB8 encodes an R2R3 MYB transcription factor which represses lignin and flavonoid synthesis in chrysanthemum. Plant Physiol. Biochem. 149, 217-224 (2020).

10. Yang, C. Study on the Mechanism of Regulating Tomato Lignin Synthesis [D] (Huazhong Agricultural Univ., 2019).

11. Cavallini, E. et al. Functional diversification of grapevine MYB5a and MYB5b in the control of flavonoid biosynthesis in a petunia anthocyanin regulatory mutant[J]. Plant Cell Physiol. 55, 517-534 (2014).

12. Li, M. et al. PpNAC187 enhances lignin synthesis in Whangkeumbae' Pear (Pyrus pyrifolia) 'hard-end' fruit. Molecules 24, 4338 (2019).
13. Yang, Y. et al. PdWND3A, a wood-associated NAC domain-containing protein, affects lignin biosynthesis and composition in Populus. BMC Plant Biol. 19, 486 (2019).

14. Mccarthy, R. L. et al. The poplar MYB transcription factors, PtrMYB3 and PtrMYB20, are involved in the regulation of secondary wall biosynthesis. Plant Cell Physiol. 51, 1084-1090 (2010).

15. Zhong, R., Richardson, E. A. \& Ye, Z. H. The MYB46 transcription factor is a direct target of SND1 and regulates secondary wall biosynthesis in Arabidopsis. Plant Cell 19, 2776-2792 (2007).

16. Mccarthy, R. L., Zhong, R. \& Ye, Z. H. MYB83 is a direct target of SND1 and acts redundantly with MYB46 in the regulation of secondary cell wall biosynthesis in Arabidopsis. Plant Cell Physiol. 50, 1950-1964 (2009).

17. Yang, $C$. et al. Arabidopsis MYB26/MALE STERILE35 regulates secondary thickening in the endothecium and is essential for anther dehiscence. Plant Cell 19, 534-548 (2007).

18. Zhou, J. et al. MYB58 and MYB63 are transcriptional activators of the lignin biosynthetic pathway during secondary cell wall formation in Arabidopsis. Plant Cell 21, 248-266 (2009).

19. Zhong, R. et al. A battery of transcription factors involved in the regulation of secondary cell wall biosynthesis in Arabidopsis. Plant Cell 20, 2763-2782 (2008).

20. Ohman, D. et al. MYB103 is required for FERULATE-5-HYDROXYLASE expression and syringyl lignin biosynthesis in Arabidopsis stems. Plant J. 73, 63-76 (2013).

21. Wang, L. et al. R2R3-MYB transcription factor MYB6 promotes anthocyanin and proanthocyanidin biosynthesis but inhibits secondary cell wall formation in Populus tomentosa. Plant J. 99, 733-751 (2019).

22. Dou, L W. The Negative Regulational Molecular Mechanism of the Poplar Transcription Factor MYB93 Involved in Flavonoids and Lignin Biosynthesis [D] (Southwest Univ., 2019).

23. Ooka, $\mathrm{H}$. et al. Comprehensive analysis of NAC family genes in Oryza sativa and Arabidopsis thaliana. DNA Res. 10, 239-247 (2003).

24. Mitsuda, N. et al. The NAC transcription factors NST1 and NST2 of Arabidopsis regulate secondary wall thickenings and are required for anther dehiscence. Plant Cell 17, 2993-3006 (2005).

25. Ko, J. H. et al. ANAC012, a member of the plant-specific NAC transcription factor family, negatively regulates xylary fiber development in Arabidopsis thaliana. Plant J. 50, 1035-1048 (2007).

26. Ohtani, M. et al. A NAC domain protein family contributing to the regulation of wood formation in poplar. Plant J. 67, 499-512 (2011).

27. Yamaguchi, M. et al. VND-INTERACTING2, a NAC domain transcription factor, negatively regulates xylem vessel formation in Arabidopsis. Plant Cell 22, 1249-1263 (2010)

28. Piccolo, E. L. et al. Girled-induced anthocyanin accumulation in red-leafed Prunus cerasifera: effect on photosynthesis, photoprotection and sugar metabolism. Plant Sci. 294, 110456 (2020).

29. Wu, C. et al. Effect of ripening stage on physicochemical properties and antioxidant profiles of a promising table fruit "pear-jujube" (Zizyphus jujuba Mill.). Sci. Hortic. 148, 177-184 (2012).

30. Huang, J. et al. Jujube cultivar vitamin C profile and nutrient dynamics during maturation. Am. Soc. Hortic. Sci. 52, 859-867 (2017).

31. Zhao, L. Q. Effect of Organizational Structure and Material Changes on Jujube Fruit Cracking in Growth and Development Process [D] (Shanxi Agricultural Univ. 2013).

32. Li, Z. H. et al. Phenylpropanoid metabolism and pigmentation show divergent patterns between brown color and green color cottons as revealed by metabolic and gene expression analyses. J. Cotton Res. $\mathbf{3}$, 27 (2020).

33. Mahabir, S. \& Narender, S. Impact of lignification on secondary cell wall development: a review. Asian J. Plant Sci. Res 9, 30-37 (2019).

34. Rogers, L. A. \& Campbell, M. M. The genetic control of lignin deposition during plant growth and development. N. Phytol. 164, 17-30 (2004).

35. Weng, J. K. \& Chapple, C. The origin and evolution of lignin biosynthesis. $N$ Phytol. 187, 273-285 (2010).

36. Guo, D. et al. Downregulation of caffeic acid 3-O-methyltransferase and caffeoyl CoA 3-O-Methyltransferase in transgenic alfalfa: impacts on lignin structure and implications for the biosynthesis of $\mathrm{G}$ and $\mathrm{S}$ Lignin. Plant Cell 13, 73-88 (2001).

37. Takeda, Y. et al. Regulation of CONIFERALDEHYDE 5-HYDROXYLASE expression to modulate cell wall lignin structure in rice. Planta 246 337-349 (2017) 
38. Zhang, W. et al. Functional characterization of CCR in birch (Betula platyphylla $\times$ Betula pendula) through overexpression and suppression analysis. Physiol. Plant 154, 283-296 (2015).

39. Hplpin, R. W. et al. Manipulation of lignin quality by down regulation of cinnamyl alcohol dehydrogenase. Plant J. 6, 121-129 (1994).

40. Shilton, B. The effect of plant hormones on phenolic production in Kauri trees. MSc thesis, Auckland University of Technology (2017).
41. Kenneth, J. L. \& Thomas, D. S. Analysis of relative gene expression data using real-time quantitative $P C R$ and the $2-\triangle \triangle C T$ method. Methods 25, 402-408 (2001).

42. Wang, N. et al. MYB12 and MYB22 play essential roles in proanthocyanidin and flavonol synthesis in red-fleshed apple (Malus sieversii f. niedzwetzkyana). Plant J. 90, 276-292 (2017).

43. An, J. P. et al. Dynamic regulation of anthocyanin biosynthesis at different light intensities by the BT2-TCP46-MYB1 module in apple. J. Exp. Bot. 71, 3094-3109 (2020). 